\title{
La investigación cualitativa en Farmacia. Aplicación en la Atención Farmacéutica
}

\author{
Djenane Ramalho de Oliveira ${ }^{1}$, Niurka Dupotey Varela ${ }^{2 *}$ \\ ${ }^{1}$ Departamento de Farmacia Social, Universidad Federal de Minas Gerais, ${ }^{2}$ Departamento de Farmacia, \\ Universidad de Oriente
}

${ }^{*}$ Correspondencia:

N. D. Varela

Calle Victoriano Garzón \# 13 Caney

Entre Camilo Cienfuegos y el Río.

Santiago de Cuba. Cuba

CP 91400

Email: dupotey@cnt.uo.edu.cu
Se presenta desde una perspectiva teórica, el estado del arte de la metodología de investigación cualitativa en el campo de las Ciencias Farmacéuticas, haciendo especial énfasis en el área de la Atención Farmacéutica. Se exponen los presupuestos teóricos e investigaciones cualitativas más importantes realizadas en el ámbito de las ciencias de la salud y en el sector asistencial farmacéutico internacional. A partir de la información expuesta, se considera que a pesar de que, la utilización de los métodos de investigación cualitativa se ha desarrollado considerablemente en el ámbito sanitario, su aplicación en el campo de la Farmacia Asistencial y de la Atención Farmacéutica, aún es insuficiente, lo que trasciende a Latinoamérica, donde el ejercicio de esta nueva filosofía de práctica profesional no constituye actualmente una actividad globalmente establecida. La evaluación desde una perspectiva social del status de la profesión farmacéutica, a partir de la percepción de pacientes, farmacéuticos y otros profesionales sanitarios, así como la profundización desde el punto de vista cualitativo, de las principales barreras que limitan la generalización de la Atención Farmacéutica, pudieran contribuir fuertemente al mejoramiento de la actual actividad farmacéutica clínica, por un uso más racional de los medicamentos y calidad de vida superior en los pacientes.
Unitermos

- Farmacia asistencial

- Atención Farmacéutica

- Pratica Farmacéutica

- Ciências Farmacêuticas/ investigaciones cualitativas

- Asistencia Farmacéutica

\section{SITUACIÓN ACTUAL Y PERSPECTIVAS DE LA PROFESIÓN FARMACÉUTICA EN EL SISTEMA DE ATENCIÓN A LA SALUD.}

La Farmacia es una profesión sanitaria asistencial que forma parte del conjunto de aquellas que atienden a las necesidades de salud de la población (Consull catalá d' especialitats en siencies de la salut, 2001).

En el actual estado de desarrollo que la medicina ofrece en estos días a profesionales y pacientes, los medicamentos son productos que se han mostrado capaces de prevenir, curar y/o mejorar numerosas enfermedades, sus síntomas y sus consecuencias y que su consumo y utilización no es siempre inocuo y a veces también presenta problemas de efectividad y seguridad, lo cual hace necesario su vigilancia (Martínez, Baena, 2001). Precisamente a esta necesidad social, responde la Farmacia como una profesión universal, integrada en el campo de la salud 
y cuya misión específica es liderar el uso correcto de los medicamentos por la sociedad (Ronda, 2006).

Hoy día existe un constante debate sobre los roles, responsabilidades y ética de las diferentes profesiones sanitarias. Es precisamente dentro de este contexto donde los farmacéuticos deben asumir su parte de responsabilidad dentro del Sistema de Salud, en todo lo que se refiere a la farmacoterapia y a través de la Atención Farmacéutica dar respuesta a la actual necesidad social de su mejor control, siendo el farmacéutico el profesional idóneo para hacerlo (Hepler, 1997).

El papel del farmacéutico en el sistema de atención a la salud, ha sido analizado en diversos foros de carácter global y desde la Declaración de Alma-Ata de Salud para todos en el año 2000, realizada en 1990, la Declaración de Tokio (Organización Panamericana de la Salud, 1993), hasta la más reciente emitida que plantea la Estrategia Farmacéutica para los años 2004-2007 (Organización Mundial de la Salud, 2004), se ha insistido en que el farmacéutico se comprometa activamente con la estrategia de atención primaria de salud, así como consolide su posición de especialista de los medicamentos en el equipo de salud, a través del ejercicio de la Atención Farmacéutica, nueva filosofía de práctica profesional donde el paciente es el principal beneficiario de las acciones del farmacéutico (Hepler, Strand, 1990).

Sin embargo, aún el rol del farmacéutico en el ámbito asistencial, no satisface las necesidades de una sociedad consumidora de medicamentos, lo que ha conducido en los últimos años a la incidencia de errores de medicación, que han llevado tanto a muertes de pacientes y a hospitalizaciones (Hepler, Segal, 2003). Existe actualmente un nefasto problema en la asistencia sanitaria que requiere urgentemente atención experta, el de la prevención de la morbi-mortalidad relacionada con los medicamentos (Hepler, Strand, 1990). Es cuando se vuelve esencialmente importante, conocer la percepción social de pacientes y profesionales de la salud sobre el desempeño clínico de los profesionales farmacéuticos, y mostrar desde una perspectiva cualitativa las representaciones de estos grupos de personas en torno a esta problemática.

\section{EL ENFOQUE CUALITATIVO DE LA INVESTIGACIÓN EN CIENCIAS DE LA SALUD}

La investigación en el campo de la salud se ha basado mayoritariamente en estudios de tipo cuantitativo, con el objetivo de medir o cuantificar de forma objetiva hechos basados en una hipótesis concreta. No obstante y debido a la necesidad de dar respuesta a nuevas preguntas generadas en la práctica clínica, se ha producido la paulatina introducción de las técnicas cualitativas en el ámbito de las Ciencias de la Salud, precisamente para responder a determinadas cuestiones que las técnicas cuantitativas no responden adecuadamente (Lorenzo, Mira, 2006; Amescua, Gálvez, 2002).

La Metodología Cualitativa se refiere a la investigación que produce datos descriptivos: las propias palabras de las personas, habladas o escritas, y la conducta observable (Rodríguez, Gil, García, 2004). Es inductiva porque los investigadores comienzan sus estudios con interrogantes vagamente formuladas y con un diseño de investigación flexible. Es holística, puesto que las personas, los escenarios o los grupos no son reducidos a variables, sino considerados como un todo. Es humanista, pues comprende a las personas dentro del marco de referencia de ellas mismas, el investigador se aparta de sus propias creencias, perspectivas, predisposiciones y todas las perspectivas, escenarios y personas son consideradas valiosas. El investigador cualitativo da énfasis a la validez de la investigación (Calderón, 2002). Aunque existen diversas técnicas para la obtención de datos en la investigación cualitativa, las prácticas más representativas de la perspectiva metodológica cualitativa de investigación social son precisamente: la observación participante, la entrevista en profundidad y los grupos de discusión (Callejo, 2002).

La promoción de la utilización de las técnicas cualitativas en el ámbito de la salud en el mundo comenzó a desarrollarse recientemente, en la década del 90. Los países que constituyen punta de lanza en su aplicación son España y los de habla anglosajona (Rodríguez, Gil, García, 2004). Apenas hasta el momento se va extendiendo a los países periféricos, éste es el caso de los países latinoamericanos. Argentina, Brasil, y México parecen ser los países de la región con la infraestructura más consolidada en este sentido (Mercado, 2002).

Esta ha contribuido en el ámbito sanitario al estudio de los determinantes de la salud y la enfermedad, a la obtención de representaciones sociales sobre la salud, la planificación sanitaria, las relaciones entre las personas enfermas y los profesionales sanitarios que las atienden, la aceptación por los sanitarios de nuevas formas de gestión, la percepción del riesgo, etc. Permite además, la detección de las necesidades, la evaluación de las intervenciones para la salud y conlleva a una comprensión más global de la concepción cultural y simbólica que implica la vivencia de la salud por los individuos y las sociedades (Conde, Pérez, 1995; Pérez, 2002).

\section{APLICACIÓN DE LA METODOLOGÍA DE INVESTIGACIÓN CUALITATIVA EN FARMACIA}

En gran medida la práctica del profesional farmacéutico está dirigida principalmente al ser humano, 
referidos como consumidores o pacientes, cada uno conectados a sus familias, organizaciones y sistemas de salud y diferentes culturas por todo el mundo, por lo que para llevar a cabo una correcta práctica farmacéutica, se necesita del complemento de disciplinas que estudien a las personas y sus sistemas de sociedad. Este es el punto donde la Farmacia Social entra en escena, en la que los medicamentos son estudiados desde la perspectiva de las ciencias sociales y humanísticas y donde se estudian creencias, actitudes, reglas y procesos relacionados con los medicamentos y la salud (Acción Internacional para la Salud, 2003).

A pesar de la extensión que a nivel internacional va experimentando la utilización de la metodología cualitativa y teniendo en cuenta que su impacto en el ámbito sanitario ha conducido definitivamente, a una forma diferente de hacer ciencia, donde se profundiza en los aspectos sociales relacionados con la salud, se considera que las investigaciones cualitativas en el sector farmacéutico son escasas, aunque sobresalen experiencias alentadoras e importantes principalmente en el Reino Unido (Akiya, Anderson, Avery, 2002; Hughes, Mccann, 2002; Tulip et al., 2002) a las cuales se hará referencia posteriormente.

La gran generalidad de los estudios que se realizan actualmente en el campo de la Farmacia Asistencial, responden al enfoque cuantitativo o positivista, lo que propicia que las investigaciones realizadas, carezcan del análisis de fenómenos relacionados con la profesión farmacéutica desde una perspectiva cualitativa, considerando la percepción, actitudes y expectativas de profesionales farmacéuticos, sanitarios y pacientes. De hecho, es importante destacar que en una búsqueda realizada recientemente (20/5/07) en MEdLine para los últimos 10 años, sólo 36 referencias respondieron a las palabras claves qualitative research or qualitative methodology in Pharmacy versus 3869 investigaciones cuantitativas.

A continuación se muestran experiencias realizadas en los últimos años en esta temática, entre las cuales se destacan las realizadas por grupos de investigación de Facultades de Farmacia del Reino Unido y Estados Unidos. Entre ellos se encuentra un trabajo realizado en Nottingham, dirigido a describir los puntos de vista de farmacéuticos y personal de enfermería de una casa de enfermeras para la atención comunitaria, en relación al rol de farmacéuticos comunitarios del área, aplicando entrevistas en profundidad a 10 enfermeras y 8 farmacéuticos y los resultados evidenciaron diferencias en la apreciación por ambas profesiones de las funciones del farmacéutico comunitario (Akiya, Anderson, Avery, 2002).

Una investigación realizada por la Sociedad Farmacéutica Real de Gran Bretaña, (The Royal Pharmaceutical Society of Great Britain), evaluó el papel de los farmacéuticos en el tratamiento de la depresión en la atención primaria. Se entrevistaron a 51 pacientes con diagnóstico de enfermedad mental, consumidores de antidepresivos y se evidenciaron dificultades en la provisión de información por los farmacéuticos a los pacientes, en relación a la medicación antidepresiva al comienzo del tratamiento, a la no adherencia, reacciones adversas, dosificación, etc. Específicamente se detectó que los pacientes no estaban informados sobre los elementos más importantes relacionados con su farmacoterapia antidepresiva (Garfield, Smith, Francis, 2002).

Un estudio realizado en Irlanda del Norte, Reino Unido, exploró e identificó a través de una investigación cualitativa las barreras percibidas por farmacéuticos comunitarios y los médicos generales, en su trabajo interprofesional. Se seleccionaron tres áreas locales de servicios de salud y se conformaron 6 grupos focales, 2 grupos por localidad. Se realizaron los grupos de discusión correspondientes y se obtuvo que los 4 temas principales considerados por ambos profesionales como barreras en la relación farmacéutico comunitario-médico fueron: la imagen del farmacéutico como tendero, la accesibilidad entre ambos profesionales, el estado del conocimiento del farmacéutico para intercambiar con el facultativo y la jerarquía de éste sobre el farmacéutico como profesional sanitario (Hugues, Mccann, 2002).

Otro estudio realizado en Londres, investigó las necesidades de información en materia de medicamentos, de 299 pacientes hospitalizados en tres hospitales docentes, a través de la combinación de técnicas cuantitativas y cualitativas, utilizando cuestionarios y entrevistas en profundidad (Astrom et al., 2000).

Estudios cualitativos en Estados Unidos reportan la utilización de las técnicas cualitativas para comprender las percepciones de los integrantes de un equipo de salud, en relación a su rol (Rodehorst, Wilhelm, Jensen, 2005), determinar las perspectivas y necesidades de información de pacientes con asma bronquial, (Raynor et al., 2004), evaluar la percepción de profesionales y administradores de una institución hospitalaria en relación a los programas diseñados para identificar errores de medicación (Tamuz, Thomas, Franchois, 2004), conocer la percepción de farmacéuticos en relación a los factores asociados a la venta de jeringas a una comunidad de Georgia (Taussig et al., 2002), determinar las barreras que limitan la adherencia al tratamiento con antirretrovirales en pacientes con VIHSIDA (Proctor, Tesfa, Tompkins, 1999), evaluar las actitudes de las madres de pacientes pediátricos hacia los farmacéuticos comunitarios (Hodgson, Wong, 2004), el impacto de un programa de educación sanitaria para el tratamiento de las diarreas (Ross-Degnan et al., 1996), los 
puntos de vistas de farmacéuticos, médicos y consumidores en relación a las necesidades de educación de los pacientes, así como las barreras de comunicación entre ellos (Ruth, Hodge, Murphy, 1994).

Igualmente se realizó un estudio en Dinamarca sobre los puntos de vista y percepciones de mujeres jóvenes de 21-34 años en relación a su utilización de inhibidores de la recaptación de serotonina (Knudsen, Hansen, Eskildsen, 2003). Otro estudio realizado igualmente en ese país, valoró las percepciones de 10 grupos sociales diferentes en relación al rol del farmacéutico comunitario en el presente y en el futuro (Norgaard, Colberg, Niemann, 2001).

En Brasil, se determinaron representaciones relacionadas con la práctica de la automedicación, en un pequeño grupo de habitantes de Minas Gerais, (Loyola, Lima-Costa, Ochoa, 2004). Igualmente un estudio fenomenológico realizado en Suecia, evaluó la experiencia de un equipo de salud en relación a la descentralización del Servicio de Farmacia, en una unidad satélite de una clínica de pacientes con VIH-SIDA, a través de entrevistas en profundidad realizadas a doctores y enfermeras (Sodergard et al., 2005).

También se reporta un estudio realizado en Viet Nam, el cual hace un análisis fenomenológico y de contenido para explorar los puntos de vistas de profesionales de la salud y pacientes en relación al rol del personal y de los servicios farmacéuticos de Hanoi, a través de 21 entrevistas en profundidad realizadas a farmacéuticos, médicos, estudiantes de farmacia y pacientes, las cuales fueron audiograbadas y transcriptas. De forma general se obtuvo que las percepciones expuestas en relación al farmacéutico, lo ubican como consejero, asistente del médico y comerciante (Olsson et al., 2002)

Un estudio realizado en Turquía, evaluó a través de la combinación de técnicas de investigación cualitativa y cuantitativa el hábito de fumar en estudiantes de farmacia desde el primero hasta el último año y se identificaron los factores asociados a este hábito tóxico. Fueron usadas las técnicas de discusión en grupo, en el desarrollo de la fase cualitativa de investigación (Yegenoglu et al., 2006)

Recientemente en Bristol, Reino Unido, se refiere una investigación que utiliza métodos cualitativos para examinar problemas relacionados con medicamentos desde la perspectiva de pacientes con enfermedades crónicas (Gordon, Smith, Dhillon, 2007).

Como puede observarse en el $100 \%$ de los estudios referidos, se hace uso de la entrevista en profundidad y grupos de discusión, para la obtención de la información cualitativa, de las cuales predominan la primera técnica en el $88,23 \%$ de los reportes mostrados. Es importante destacar que dentro de los estudios que se refieren, la evaluación de la percepción, actitudes y comportamientos de los farmacéuticos, en relación a las funciones profesionales, ocupa el 41,17\%, lo que evidencia que dentro del campo de las funciones de la profesión, existe una urgente necesidad de continuar implementando investigaciones cualitativas, que permitan, a través de las perspectivas sociales de farmacéuticos, pacientes, médicos y enfermeras, esclarecer el status de la profesión farmacéutica en toda su extensión, para que se promuevan las intervenciones pertinentes y el anhelado reconocimiento social de la profesión farmacéutica se concrete.

\section{METODOLOGÍA DE INVESTIGACIÓN CUALITATIVA EN EL CAMPO ESPECIAL DE LA ATENCIÓN FARMACÉUTICA}

La existencia actual de Problemas Relacionados a los Medicamentos (PRM) en la atención sanitaria, así como de la propia metodología que propone la Atención Farmacéutica para identificarlos, resolverlos y prevenirlos, ahora vistos como elementos de proceso y causas de Resultados Negativos asociados a la Medicación (RNM), por el Tercer Consenso de Granada, (Comité de Consenso de Granada, 2007), alerta sobre el significado de que aún no sea esta práctica profesional establecida en la relación farmacéutico-paciente.

A pesar de los constatados beneficios humanísticos, clínicos y económicos de programas de Atención Farmacéutica que se han desarrollado en Europa, (Álvarez de Toledo et al., 2001; Martin-Calero et al., 2004; Berenguer et al., 2004), Estados Unidos (Strand et al., 2004; AssaEley, Kimberlin, 2005), y de los avances que actualmente se experimentan en algunos países latinoamericanos entre ellos Brasil, aún ésta no constituye una práctica habitualmente realizada por los farmacéuticos, su ejercicio aún no se ha implementado globalmente en muchos sistemas de salud, siendo limitado y no sistemático.

Por otro lado, muchos de estos sistemas de salud intentan aproximarse a la implantación de la Atención Farmacéutica, poniendo en marcha programas experimentales a nivel comunitario y hospitalario, sin identificar socialmente aquellas barreras que desde la perspectiva de los pacientes, farmacéuticos, profesionales de la salud, y sistema de salud en general, limitan el desarrollo de este modelo de práctica profesional, así como otros aspectos que en el entorno psicosocial se manifiestan y caracterizan la práctica profesional de los farmacéuticos.

De modo que la problemática planteada, se precisa en la ausencia de estudios que desde una perspectiva social, determinen el status de la práctica clínica farmacéutica, como justificación para la adopción de la Atención Farmacéutica como nuevo modelo de práctica profesional 
en los servicios farmacéuticos asistenciales. América Latina y Cuba en particular no escapan a esta problemática y los servicios farmacéuticos asistenciales enfrentan un ejercicio de funciones clínicas limitado en relación al desempeño de otras actividades como las comerciales, administrativas y de dirección, enfocándose aún la actividad más al medicamento que al propio paciente.

Según una investigación realizada por la Pharmaceutical Care Network Europe (PCNE) (Foop, 2000), se han identificado barreras en las que se incluyen precisamente el estado de la relación farmacéutico-paciente y farmacéutico-profesionales sanitarios, elementos con los que se concuerda y lo que debiera ser objeto de evaluación a través de la realización de investigaciones que respondan al paradigma metodológico interpretativo o cualitativo.

Sin embargo, la incursión en las metodologías cualitativas para evaluar las percepciones, representaciones, actitudes y comportamientos tanto de pacientes como de profesionales en torno a la Atención Farmacéutica, es a nivel internacional bastante limitada. A esta situación se relaciona la insuficiente inclusión en el currículo del profesional farmacéutico, de elementos metodológicos que refuercen la aplicación de estas técnicas, en la práctica y la investigación farmacéutica.

Una de las investigaciones realizadas en esta dirección, fue centrada hace pocos años por el Servicio Nacional de Salud del Reino Unido, para describir el estado del cambio de la práctica farmacéutica tradicional a la Atención Farmacéutica, haciendo énfasis en los factores que pudieran constituir barreras para la implementación de un modelo estructurado de esta nueva filosofía de práctica profesional. Para ellos se realizaron grupos focales y entrevistas en profundidad para conocer los puntos de vista y percepciones de los profesionales del equipo de salud. Se realizaron 4 grupos focales con los profesionales farmacéuticos, los que expusieron sus puntos de vista en relación con la práctica farmacéutica corriente, con el modelo propuesto de atención farmacéutica y las barreras posibles, sentimientos acerca de sus roles, entrenamientos y habilidades. Se realizaron 12 entrevistas en profundidad con el personal de enfermería y doctores. Se demostró de forma general consenso entre todos los profesionales de la necesidad de implementación de la Atención Farmacéutica para aumentar la calidad de la asistencia proporcionada a los pacientes, aprobándose el modelo propuesto, siendo la principal barrera la falta de recursos, aunque también se evidenció la presencia de barreras culturales (Tulip et al., 2002).

Otra investigación fue realizada en el Reino Unido, para evaluar la percepción de profesionales de la salud y de pacientes hospitalizados con artritis reumatoide, relacionada con las necesidades de estos últimos de Atención
Farmacéutica, a través de entrevistas semi-estructuradas y grupos de discusión. Los principales puntos de vista estuvieron asociados a la necesidad del control del dolor, de la necesidad de una mayor intervención e integración del farmacéutico al equipo asistencial para el mejor uso de los medicamentos (O'hare et al., 2001).

En los Estados Unidos se realizó un estudio etnográfico de la práctica de Atención Farmacéutica por un periodo de 8 meses (Ramalho de Oliveira, 2003). En este estudio, fueran utilizadas varias técnicas de recolección de datos, entre ellas, la observación participante en clínicas donde son ofrecidos servicios de Atención Farmacéutica, entrevistas en profundidad, grupos focales y análisis de documentos. Los resultados revelan notables narraciones e impresiones de pacientes, farmacéuticos y estudiantes de Farmacia, sobre sus sentimientos y experiencias con esta nueva práctica profesional. Se evidenció según la percepción de los pacientes, la necesidad de que el farmacéutico considere al paciente de forma individual, y los farmacéuticos reconocieron que su actitud frente a los pacientes afecta realmente los resultados de esa atención que se les provee (Ramalho de Oliveira, Shoemaker, 2006).

Es importante destacar los estudios que relacionados con la Atención Farmacéutica se realizan en Brasil. Un ejemplo de esto, es una investigación fenomenológica basada en la hermenéutica, realizada por profesionales farmacéuticos de la Escuela de Farmacia de la Universidad Federal de Minas Gerais, Belo Horizonte, en torno a la percepción de los beneficios que reporta la Atención Farmacéutica para los pacientes. Los métodos utilizados fueron la observación participante y entrevistas en profundidad con pacientes y farmacéuticos (Freitas, 2005; Pereira, 2006). Es importante apuntar que se evidenciaron resultados semejantes a los obtenidos en los estudios realizados en los Estados Unidos, lo que pudiera estar relacionado con el hecho de que los servicios de Atención Farmacéutica ofrecidos en los Estados Unidos y en Brasil tienen la misma filosofía de práctica y utilizan el mismo proceso de cuidado del paciente. Los pacientes se sienten a gusto teniendo un profesional de la salud responsable por la gerencia de su farmacoterapia y los farmacéuticos se sienten valorados como profesional de la salud.

Recientemente en Brasil fue celebrado el 1er Taller Internacional para la implantación de la Atención Farmacéutica en el Sistema de Salud, del 24 al 27 de Mayo del 2006. Este evento propició la presentación de serias investigaciones que en esa región se realizan utilizando la metodología de investigación cualitativa, precisamente para describir las percepciones y vivencias de profesionales farmacéuticos que practican la Atención Farmacéutica y de equipos de salud involucrados en programas de este tipo, 
a través de la instrumentación de la observación participante $y$ entrevistas en profundidad (Pereira, Ramalho de Oliveira, 2006; Ramalho de Oliveira et al., 2006)

Reportes más recientes, refieren la utilización de estas técnicas para describir la percepción de pacientes, en relación al significado de sus medicaciones (Shoemaker, Ramalho de Oliveira, 2008), e igualmente para mostrar la relación farmacéutico-paciente desde el punto de vista de ambas partes, teniendo en cuenta actitudes y responsabilidades (Worley et al., 2007).

En Cuba, se han realizado recientemente estudios de investigación para la implantación de programas de Atención Farmacéutica en diversos servicios comunitarios y hospitalarios, sin embargo aún no se han identificado las barreras que a nivel social limitan la generalización de esta práctica profesional, partiendo de las percepciones de pacientes, farmacéuticos y otros profesionales de la salud. Actualmente se desarrolla un proyecto de investigación en Santiago de Cuba, segunda ciudad más importante del país, con el objetivo de establecer una estrategia para la implantación de la Atención Farmacéutica en el territorio, a partir de la percepción y actitudes de pacientes, farmacéuticos, directivos y sanitarios en general. En el proyecto citado se combinan las metodologías cuantitativas y cualitativas, para determinar cómo los diferentes grupos sociales vinculados a la práctica farmacéutica, perciben a los profesionales farmacéuticos, haciendo énfasis en los diferentes servicios clínicos prestados, su grado de satisfacción con éstos, las principales actividades realizadas con el equipo de salud, desde la perspectiva de todos los involucrados, etc.

De forma general, en un importante número de investigaciones en Atención Farmacéutica está representada fuertemente la metodología de investigación cuantitativa (Mobach, 2002). De hecho, en revisión realizada recientemente (20/5/07), en la base MedLine para la elaboración de la revisión presentada, sólo se obtuvieron en los últimos 10 años, 16 referencias de estudios, bajo las palabras claves: qualitative methodology or qualitative research in Pharmaceutical Care, versus 2544 investigaciones cuantitativas.

En el $100 \%$ de estos estudios se utiliza la combinación de la entrevista en profundidad y los grupos de discusión para la obtención de la información. Se plantea que aunque éstos son considerados de gran relevancia para el avance de la Atención Farmacéutica, aún es insuficiente la aplicación de la metodología cualitativa en esta temática para la actual profesión, lo que corrobora la necesidad de instrumentar investigaciones en este sentido para lograr la implantación y generalización de esta filosofía de práctica profesional.

\section{CONCLUSIONES}

A partir del análisis realizado anteriormente se evidencia lo siguiente:

- La necesidad de perfeccionar la Farmacia Social como área interdisciplinaria en los currículos para la formación de profesionales farmacéuticos, disciplina que ofrezca las herramientas precisas a través del conocimiento de teorías y métodos de las Ciencias Sociales y Humanísticas, para investigar los aspectos psicosociales relacionados a la farmacoterapia, así como, en la evaluación de la relación y comunicación existentes entre pacientes y profesionales sanitarios.

- La metodología de investigación cualitativa y su aplicación en las Ciencias de la Salud, aporta una importante herramienta para abrir el análisis de problemáticas sanitarias desde una perspectiva social y cultural que supere los marcos estrechos proporcionados por la cuantificación estadística, profundizando en las actitudes, percepciones, comportamientos, creencias y representaciones sociales relacionadas a fenómenos del ámbito sanitario, desde el punto de vista de los profesionales hasta el de los pacientes.

- La escasa aplicación de la metodología cualitativa en el ejercicio e investigación de la Atención Farmacéutica, y la necesidad de desarrollar programas que a través del uso de estas técnicas, contribuyan a evaluar más integralmente los beneficios clínicos y humanísticos de este nuevo modelo de práctica profesional.

De hecho el desarrollo de investigaciones en el campo de la Farmacia Asistencial utilizando la metodología cualitativa en combinación con las ya aplicadas técnicas cuantitativas, contribuirá en un futuro a obtener definitivamente la armonía social y el reconocimiento que necesita el profesional farmacéutico como especialista de los medicamentos. Aun más importante, la utilización de las técnicas cualitativas de investigación permitirá conocer a los pacientes más profundamente, revelando sus deseos, necesidades, expectativas e inquietudes relacionadas al uso de los medicamentos. Así se podrá tener verdaderamente al ser humano como el centro de la práctica profesional farmacéutica y asegurar el uso más efectivo y seguro de medicamentos en la sociedad.

\section{RESUMO}

\section{A investigação qualitativa em Farmácia: Aplicação em Atenção Farmacêutica}

O estado da arte da metodologia de investigação qualitativa no campo das Ciências Farmacêuticas é apresentado neste artigo, dando ênfase especial à área da Atenção Far- 
macêutica. Apresentamos os pressupostos teóricos e as investigações qualitativas mais importantes realizadas na área das ciências da saúde e no setor farmacêutico internacional. A partir da informação exposta, observamos que apesar do uso considerável dos métodos de investigação qualitativa no ambiente sanitário, sua aplicação no campo da Farmácia Assistencial e da Atenção Farmacêutica é ainda insuficiente, o que parece transcender para a América Latina, na qual o exercício desta nova filosofia de prática profissional ainda não se apresenta como atividade amplamente estabelecida. A avaliação do estado da profissãofarmacêutica sob perspectiva social, a partir da percepção de pacientes, farmacêuticos e outros profissionais sanitários, assim como compreensão mais aprofundada dos principais desafios para a implementação da Atenção Farmacêutica, do ponto de vista qualitativo, podem contribuir fortemente para o avanço das atividades farmacêuticas clinicas, e, conseqüentemente, para o uso mais racional dos medicamentos para qualidade de vida dos pacientes.

UNITERMOS: Farmácia Assistencial. Atenção Farmacêutica. Prática farmacêutica. Ciências Farmacêuticas/ pesquisa qualitativa. Assistência Farmacêutica.

\section{REFERÊNCIAS BIBLIOGRÁFICAS}

ACCIÓN INTERNACIONAL PARA LA SALUD (AIS). ¿Farmacia Social? The Cronic ill. Disponible en: $<$ http:/ / www.aislac.org>. Acceso en: 20 Enero 2006.

AKIYA, Y.; ANDERSON, C.; AVERY, A. Community pharmacist's roles: nursing home staff and community pharmacist's views. In J Pharm Pract., v.10, p.32, 2002. Disponible en: $<$ http://www.pjonline.com/IJPP/bpc2002/ ijpp_bpc2002_r32.pdf > . Acceso en: 20 enero 2006.

ÁLVAREZ DE TOLEDO, F.; ARCOS, P.; EYARALAR, T.; ABAL, F.; DAGO, A.; CABIEDES, L.; SÁNCHEZ, I. Atención Farmacéutica en personas que han sufrido episodios coronarios agudos. Rev. Esp. Salud Pública, v.75, n.4, p.375-388, 2001.

AMEZCUA, M.; GÁLVEZ, A. Los modos de análisis en investigación cualitativa en salud: perspectiva crítica y reflexiones en voz alta. Rev. Esp. Salud Pública, v.76, n.5, p.423-426, 2002.

ASSA-ELEY, M.; KIMBERLIN, C. L. Using interpersonal perception to characterize pharmacists' and patients' perceptions of the benefits of pharmaceutical care. Health Commun., v.17, n.1, p.41-56, 2005.
ASTROM, K.; CARLSSON, J.; BATES, I.; WEBB, D. G.; DUGGAN, C.; SANGHANI, P.; MCROBBIE, D. Desire for information about drugs. A multi-method study in general medical inpatients. Pharm. World Sci., v.22, n.4, p.159-164, 2000.

BERENGUER, B.; LA CASA, C.; DE LA MATTA, M. J.; MARTIN-CALERO, M. J. Pharmaceutical care: past, present and future. Curr. Pharm. Des., v.10, n.31, p.39313946,2004.

CALDERÓN, C. Criterios de calidad en la investigación cualitativa en salud: apuntes para un debate necesario. Rev. Esp. Salud Pública, v.76, n.5, p.473-482, 2002.

CALLEJO, J. Observación, entrevista y grupo de discusión: el silencio de las tres prácticas de investigación. Rev. Esp. Salud Pública, v.76, n.5, p.409-422, 2002.

COMITÉ DE CONSENSO DE GRANADA. Tercer Consenso de Granada, sobre Problemas Relacionados con Medicamentos (PRM) y Resultados Negativos de la Medicación(RNM). Ars Pharm., v.48, n.1, p.5-17, 2007.

CONDE, F.; PÉREZ,C. La investigación cualitativa en salud pública. Rev. Esp. Salud Pública., v.69, n.1, p.145-149, 1995.

FOOP, J.W. Atención Farmacéutica en Farmacia Comunitaria en Europa, retos y barreras. Pharm Care Esp., v.2, n.1, p.42-56, 2000.

FREITAS, E. L. Revelando a experiência do paciente com a prática da atenção farmacêutica: uma abordagem qualitativa. Minas Gerais, 2005. 98p. [Tesis de Maestría en Ciencias Farmacéuticas. Facultad de Farmacia. Universidad Federal de Minas Gerais].

GARFIELD, S.; SMITH, F.J.; FRANCIS, S.A. Roles for pharmacists in the treatment of depression in primary care. Int. J. Pharm. Pract., v.10, p.59, 2002. Disponible en: $<$ http://www.pjonline.com/IJPP/bpc2002/ ijpp_bpc2002_r59.pdf $>$. Acceso en: 20 enero 2006.

GORDON, K.; SMITH, F.; DHILLON, S. Effective chronic disease management: Patients' perspectives on medication-related problems. Patient Education and Counselin.,v.65, n.3, p.407-441, 2007. 
HEPLER, C. H. Práctica y formación farmacéutica para el 2010. Farmacia Clínica, v.14, n.2, p.27-146, 1997.

HEPLER, C. H.; SEGAL, R. Preventing, medication errors and improving drug therapy outcomes. A management systems approach. Florida: CRC Press LLC, 2003. p.13,77.

HEPLER, C. H.; STRAND, L. Oportunidades y responsabilidades en la Atención Farmacéutica. Pharm. Care Esp., v.1, n.1, p.35-47, 1999.

HODGSON, C.; WONG, I. What do mothers of young children think of community pharmacists? A descriptive survey. J. Fam. Health Care, v.14, n.3, p.73-74, 76-79, 2004.

HUGUES, C.; MCCANN, S. A. qualitative assessment of perceived barriers to inter-professional working between community pharmacists and general practitioners. In J Pharm Pract., v.10, p.36, 2002. Disponible en: $<$ http:// w w w.pjon 1 in e.com/ I J P / b p c 2002 / ijpp_bpc2002_r36.pdf>. Acceso en: 20 enero 2006.

KNUDSEN, P.; HANSEN, E. H.; ESKILDSEN, K. Leading ordinary lives: a qualitative study of younger women's perceived functions of antidepressants. Pharm. World Sci., v.25, n.4, p.162-167, 2003.

LORENZO, S.; MIRA, J. La Técnica Delphi de investigación cualitativa. Disponible en: $<\mathrm{http}$ :www. aecirujanos.es/ secciones/gestión de calidad/cap4.pdf $>$. Acceso en: 15 Enero 2006.

LOYOLA, A.; LIMA-COSTA, M.; UCHOA, E. Bambui Project: a qualitative approach to self-medication. $\mathrm{Cad}$. Saude Public., v.20, n.6, p.1661-1669, 2004.

MARTIN-CALERO, M. J.;MACHUCA, M.;MURILLO, M. D.; CANSINO, J.; GASTELURRUTIA, M. A; FAUS, M.J. Structural process and implementation programs of pharmaceutical care in different countries. Curr. Pharm. Des., v.10, n.31, p.3969-3985,2004.

MARTÍNEZ, J.; BAENA, M. I. La Atención Farmacéutica como método para mejorar la salud de los pacientes y la coordinación entre los profesionales médicos y farmacéuticos (Contestación a Enrique Costas Lombardía). Pharm. Care Esp.,v.3, n.2, p.135-139, 2001.
MERCADO, F.;VILLASEÑOR, M.; LIZARDI, A. Situación actual de la investigación cualitativa en salud. Un campo en consolidación.Revista de la Universidad de Guadalajara. Dossier Investigación Cualitativa en Salud, n.17. 2000. Disponible en: http:/www.cge.udg.mx/ revistaudg/rug17/1situacion.html. Acceso en: 16 mar. 2006

MERCADO, F. Investigación cualitativa en América Latina: perspectivas críticas en salud. Int.J. Qual. Meth., v.1, n.1, p.1-27, 2002.

MOBACH, M. P. Pharmaceutical care research: hard or soft?. Part II. Pharm World Sci., v.24, n.1, p.1-4, 2002.

NORGAARD, L. S.; COLBERG, L.; NIEMANN, M. R. The role of the Danish community pharmacist: perceptions and future scenarios. Pharm World Sci., v.23, n.4, p.159-164, 2001.

O'HARE, R.; MUIR, A.; CHAPMAN, S.; WATSON, A.; HUDSON, S. A. Identification of the pharmaceutical care issues of rheumatoid arthritis patients in secondary care. Pharm. World Sci., v.23, n.5, p.183-184, 2001.

OLSSON, E.; TUYET, L. T.; NGUYEN, H. A.; STALSB, Y.; LUNDBORG, C. Health professionals' and consumers' views on the role of the pharmacy personnel and the pharmacy service in Hanoi, Vietnam - a qualitative study. J. Clin. Pharm. Ther., v.27, n.4, p.273-280, 2002.

ORGANIZACIÓN PANAMERICANA DE LA SALUD. El papel del farmacéutico en el sistema de atención a la salud. Informe de la reunión de la OMS. Tokio, Japón, 31 de Agosto de 1993. Disponible en: <http:// www.cofybcf.org.ar/download/OMSaf.doc $>$. Acceso en: 28 jul. 2008.

ORGANIZACIÓN MUNDIAL DE LA SALUD. Estrategia Farmacéutica de la OMS 2004-2007. Lo esencial son los países: Respuesta a las necesidades de los países. Disponible en: http://www.who.int/medicinedocs/collect/ edmweb/pdf/s5522s/s5522s.pdf. Acceso en: 18 feb. 2007.

PEREIRA, M.L. ReDescobrindo a atencao farmaceutica: uma visão qualitativa da implantação de um serviço de Atenção Farmacêutica em farmácia comunitária. Belo Horizonte, 2006. 98p. [Tesis de Maestría en Ciencias Farmacéuticas. Facultad de Farmacia. Universidad Federal de Minas Gerais]. 
PEREIRA, M. L.; RAMALHO DE OLIVEIRA, D. Entendendo a implentacão de um Servico de Atenção Farmacêutica. In: SEMINÁRIO INTERNACIONAL PARA IMPLEMENTACÃO DA ATENCÃO FARMACÊUTICA, 2006. Anais. Brasília: Editora?, 2006. res. P16.

PÉREZ, C. Sobre la metodología cualitativa. Rev. Esp. Salud Pública., v.76, n.5, p.373-380, 2002.

PLENO DE “CONSULL CATALÁ D’ ESPACIALITATS EN SILENCIES DE LA SALUT" (CCECS). Competencias de la profesión de Farmacia. 2001. Disponible en: <http://www.pharmaceutical-care.org/ com_farmacias.htm>. Acceso en: 3 Mar. 2006.

PROCTOR, V.E.; TESFA, A.; TOMPKINS, D. C. Barriers to adherence to highly active antiretroviral therapy as expressed by people living with HIV/AIDS. AIDS Patient Care, v.13, n.9, p.535-544, 1999.

RAMALHO DE OLIVEIRA, D. Pharmaceutical care uncovered: an ethnographic study of pharmaceutical care practice. Minneapolis, 2003. 100p. [Tesis de Doctorado en Ciencias Farmacéuticas. Facultad de Farmacia. Universidad de Minnesota].

RAMALHO DE OLIVEIRA, D.; SHOEMAKER, S.J. Achieving patient centeredness in pharmacy practice: openness and the pharmacist's natural attitude. J. Am. Pharm. Assoc., v.46, n.1, p.56-64, 2006.

RAMALHO DE OLIVEIRA,D.;LOURENCO DE FREITAS, E.; PERINI,E.; MEDINA, S.; TACIANA, B. O Farmacêutico no PSF: Vivencias da equipe de Saude com a Atenção Farmacêutica. Anais "Semeando o cuidado ao paciente e o uso racional dos medicamentos", res. P17, 2006. (Presentado en Seminário Internacional para implementação da Atenção Farmacêutica, no SUS, 1, Brasilia-DF, 2006)

RAYNOR, D. K.; SAVAGE, I.; KNAPP, P.; HENLEY, J. We are the experts: people wit asthma talk about their medicine information needs. Patient Educ. Couns., v.53, n.2, p.167-174, 2004.

RODEHORST, T. K.; WILHELM, S. L.; JENSEN, L. Use of interdisciplinary simulation to understand perceptions of team members' roles. J. Prof. Nurs., v.21, n.3, p.59-66, 2005.
RODRÍGUEZ, G.; GIL, J.; GARCÍA, E. Metodología de la investigación cualitativa. La Habana: Editorial Félix Varela, 2004. p.39-57.

RONDA J. La Farmacia no es un comercio de "todo a cien". Disponible en: <http:/www. lists. albura.net/efe.es/ apuntes/2003-5/authors.html $>$. Acceso en: 10 Febrero 2006).

ROSS-DEGNAN, D.; SOUMERAI, S. B.; GOEL, P. K.; BATES, J.; MAKHULO, J.; DONDI, N.; SUTOTO, I.; ADI, D.; FERRAZ-TABOR, L.; HOGAN, R. The impact of face-to-face educational outreach on diarrhoea treatment in pharmacies. Health Policy Plan., v.11, n.3, p.308-18, 1996.

RUTH, D.; HODGE, M. M.; MURPHY, B. Patient drug education.Improving the working relationship between general practitioners and pharmacists. Aust. Fam. Physician., v.23, n.8, p.1536-1541, 1994.

SHOEMAKER, S. J; RAMALHO DE OLIVEIRA, D. Understanding the meaning of medications for patients: The medication experience. Pharm. World Sci., v.30, n.1, p.86-91, 2008.

SODERGARD, B. M.; BARETTA, K.; TULLY, M. P.; KETTIS, A. M. A qualitative study of health-care personnel's experience of a satellite pharmacy at a HIV clinic. Pharm. World Sci., v.27, n.2, p.108-115, 2005.

STRAND, L. M.; CIPOLLE, R. J.; MORLEY, P. C.; FRAKES, M. J.The impact of pharmaceutical care practice on the practitioner and the patient in the ambulatory practice setting: twenty-five years of experience. Curr. Pharm. Des., v.10, n.31, p.39874001,2004 .

TAMUZ, M.; THOMAS, E. J.; FRANCHOIS, K. E. Defining and classifying medical error: lessons for patient safety reporting systems. Qual Saf Health Care., v.13, n.1, p.3-20, 2004.

TAUSSIG, J.; JUNGE, B.; BURRIS, S.; JONES, T.S.; STERK, C. E. Individual and structural influences shaping pharmacists' decisions to sell syringes to injection drug users in Atlanta, Georgia. J. Am. Pharm. Assoc., v.42, n.6, p.40-45, 2002. 
TULIP, S.;CHEUNG, P.;CAMPBELL, D.; WALTERS, P.Pharmaceutical care: cultural, practice and professional perspectives. Int. J. Pharm Pract., v.10, p.93, 2002. Disponible en: $<$ http://www.pjonline.com/IJPP/bpc2002/ ijpp_bpc2002_r93.pdf $>$. Acceso en: 20 enero 2006.

WORLEY, M. M.; SCHOMMER, J. C.; BROWN, L. M.; HADSALL, R. S.; RANELLI, P. L.; STRATTON, T. P.; UDEN, D. L. Pharmacists' and patients' roles in the pharmacist-patient relationship: are pharmacists and patients reading from the same relationship script?. Res. Social Adm. Pharm., v.3, n.1, p.47-69, 2007.
YEGENOGLU, S.; ASLAN, D.; ERDENER, S. E.; ACAR, A.; BILIR, N.What is venid smoking among pharmacy students: a quantitative and qualitative study from Turkey. Subst. Use Misuse., v.41, n.3, p.405-414, 2006.

Recebido para publicação em 10 de março de 2008 Aceito para publicação em 15 de julho de 2008 